\title{
Validation of a Systematic Approach to Requirements Engineering Process Improvement in SMEs in a Design Science Framework
}

\author{
Edward Kabaale \\ Makerere University Business \\ School \\ P.O.Box 1337, Kampala, \\ Uganda
}

\author{
Christine Amulen \\ Makerere University Business \\ School \\ P.O.Box 1337, Kampala, \\ Uganda
}

\author{
Geoffrey Kituyi \\ Makerere University Business \\ School \\ P.O.Box 1337, Kampala, \\ Uganda
}

\begin{abstract}
Requirements Engineering (RE) acts as a conduit between the needs of system users and the capabilities of software produced. Because of this, many Small and Medium Enterprises (SMEs) have been tempted to invest in improving corresponding processes. Regrettably though, many process improvement approaches for RE do not meet SMEs' needs and are often hard to apply in these companies especially in transitional countries such as Uganda. This study therefore, presents a validation of a Systematic Approach to Requirements Engineering Process Improvement that can be used to enable RE process improvement in SME software companies in transitional countries. The validation was conducted following a design science research approach where four (4) case organizations were used. From the study, it is reported that the approach is indeed easy to use, understandable and is applicable to all kinds of SMEs that would wish to conduct RE process improvement.
\end{abstract}

\section{General Terms}

Requirements engineering, Process Improvement and Small and Medium Software Companies

\section{Keywords}

Requirements Engineering, Systematic Approach, SMEs, Design Science and Validation

\section{INTRODUCTION}

Recently the need to improve the requirements engineering (RE) process has been embraced. This is witnessed from the emergency of Requirements Engineering Process Improvement (REPI) and assessment models and standards such as the Good practice guide [16], Flexible and Pragmatic RE framework for SMEs [14] and Requirements-Capability Maturity Model [2] among others. However little progress has been registered in SMEs using these models in improving their RE processes. This is because SMEs find it difficult to implement REPI process improvements as they cannot bear the cost of implementation; more so the limited resources and the strict time constraints in which they operate [11]. More over SMEs have got specific problems due to their size and the budget constraints under which they operate, the maturity level in software engineering is very low, little resources to consider quality and process improvements, very few SMEs document their requirements and there are no clear ways of RE process verification and validation [9]. [20] Argues that where SMEs have in place RE process, it's always very difficult to improve such practices because it has an economic implication to the organization. Any REPI plan requires an assessment about the current status of RE process development in the software companies, and a description of the strengths and weaknesses identifying potential areas for improvement [3].

Notwithstanding, many SME software companies are interested in improving their RE processes because of their confidence that RE can be the key to developing successful software systems [11]. For instance in the survey of twelve SMEs in Finland, it is revealed that the SMEs management is not aware of the available REPI methods but there is desire to start them [13]. As such there is need to support SMEs to improve their RE processes. It is on this basis that this study attempted to validate the designed systematic approach [8] that could be used to support SME software companies to conduct their requirements engineering process improvement. Systematic process improvement is a goal-oriented measurement and controlled way of introducing process change, with predictable outcome in terms of quality, time and productivity [18] define. The approach was designed using requirements that were derived from the challenges identified in the exploratory study reported in [8]. The challenges included among others lack of user involvement, unavailability of the facilities that support process improvement efforts, lack of skills, ambiguous and changing requirements, costly process, measurement of RE process benefits, and resistance to change. These challenges if well managed can lead to better management of REPI in SMEs [10].

The requirements derived from these challenges [8] included: (i) supporting user involvement to enable the new process to succeed and be institutionalized [10]. This can be achieved through conducting an assessment of current RE process to provide information that is used to set realistic improvement goals, process improvement planning and practical actions for SMEs [10], and to guide SMEs towards optimizing their RE processes. Involving users in defining the simple processes and practice makes it very easy for users to learn and work with the new improved processes, as well as integrating new processes incrementally and gradually; (ii) using evolutionary improvement strategy by introducing small-scale improvements with a high benefit/cost ratio before expensive new techniques [16]. Moreover it will help in avoiding unnecessary project risks that may be caused by rapid changes in the organization; (iii) support change management so as to minimize employee resistance to new and improved RE processes. This can be done by adapting the new RE process, i.e. tailor the improved process to the organization. The new $\mathrm{RE}$ process should be adapted to the needs of the organization and be integrated in the daily routines of the organization; (iv) support training and education to promote the good 
understanding of the RE process to all the people involved in the improvement process. This can be enabled by creating awareness and promoting the new RE process. This involves usage of the new RE process benefits to promote its use in the organization and persuading software product development teams to adopt the new RE process and secure the support of senior management; and (v) encouraging management commitment and support to process improvement can be in form of funding, allocation of staff and providing a conducive environment for working [10]. This can be done by managers promoting systematic use of the new processes in the organization. In the section that follows we present the research approach that was followed to validate the systematic approach, while section 3 is a description of the evaluation and validation of the systematic approach to REPI in Ugandan SMEs and finally conclude with pointers to future research in section 4.

\section{RESEARCH APPROACH}

To validate the Systematic approach to REPI for SMEs, we followed the design science (DS) approach. Design science is fundamentally a problem solving paradigm and therefore seeks to produce constructs, models, methods, and instantiations [7]. The created artifacts extend the boundaries of human problem solving within organizational capabilities [7] and are tangible recommendations that enable IT researchers to tackle the problems inborn in developing and deploying information systems within organizations. The design science research method was chosen based on its ability in solving practical problems that have besieged the Information system arena for some time by creating successfully IT artifacts [15]. We specifically followed the model suggested by [15] that was tested and verified using RE case studies, the subject matter of this research [15]. This research model has six steps for conducting and evaluating good design-science research: Problem identification and motivation, objectives of a solution, design and development, demonstration and Testing, evaluation and validation and communication. In this research the identified problem was the lack of a systematic approach to enabling SMEs to conduct their REPI. The study aimed at validating a systematic approach to REPI in SMEs (SAREPI). The systematic approach was designed based on requirements derived from the exploratory study challenges and recommendations [8] and finally validated the approach using the suggested design science value parameters (usability, understandability, simplicity, completeness and applicability) [5] in four case SME organizations in Uganda. A sample of 28 out of 30 (who participated in the exploratory study) experts were involved in the validation process representing 93\% participation. No reasons were given as to why the two experts did not participate in the validation task. According to [6] and [1], small samples can be used to validate explanations and support model development.

Small samples can also be used to get expert feedback to evaluate models and approaches. This forms the basis of our motivation to use small samples to validate the designed approach to RE process improvement more especially in SMEs were there are few experts in the area. The case organizations included Makerere University Business School (Socket works project); Makerere University School of Computing and Informatics Technology (Department of Innovations and Software Development); Crystal Clear Software Ltd; and Software Factory Ltd. The group composed of requirements engineers, systems analysts, software developers as well as project managers. These were exposed to the systematic approach and were asked to evaluate it based on the set criteria given in section 4 .

\section{VALIDATION OF THE SYSTEMATIC APPROACH TO REPI (SAREPI) IN SMES}

In this section, we present and discuss the validation of the SAREPI having been exposed to four case SMEs organizations. The SAREPI is a product of the derived requirements described in section 1 . In performing the REPI, the existing generic steps suggested by [5] are followed to manage/effect the SAREPI requirements, more so they are considered important in the REPI plan as shown in figure 1.

\subsection{Validation Criteria}

An evaluation criterion was set to find out from the respondents how well the designed approach responded to their challenges: Ease of Use - The goal of this criterion was to locate areas of confusion and ambiguity for the users which, when improved increases the efficiency and quality of a users' experience with the approach. Validation factors included; the approach being simple yet retain meaning, the approach should require little or no training to be used, the approach should be easy to learn and understand and the approach should bring about user satisfaction in using it. Understandable - All users of the approach should develop a shared understanding of the RE process in order to identify where improvement is needed. There should be no ambiguity in interpretation, especially when goals are set for improvement and all terms should be clearly defined. Validation factors included; use of simple language, approach steps to follow well defined, clear definition of approach terms and no ambiguity i.e. Systematic flow of the approach. Understanding is a prerequisite for effective process improvement and management [1]. Completeness - The goal of this parameter was to find out from the respondents how well the approach covered the aspects of REPI in SMEs and their challenges. Validation factors included; scope of the approach, approach containing enough detail yet remains simple to understand and use. Applicability - This factor assessed the approach's applicability to SME software companies in the Ugandan context. To determine the approach's applicability, we used flexibility, easy to tailor to different SMEs environments and simple to adopt approach.

Table 1. Responses on SERAPI Validation

\begin{tabular}{|l|c|c|}
\hline Variables & Agree (\%) & Disagree (\%) \\
\hline Ease of Use & 79 & 21 \\
\hline Easy to understand & 80 & 20 \\
\hline Approach Completeness & 82 & 18 \\
\hline Approach applicability & 80 & 20 \\
\hline Average Percentage & $\mathbf{8 0 . 2 5}$ & $\mathbf{1 9 . 7 5}$ \\
\hline
\end{tabular}

From Table 1, results revealed that the approach is easy to use (79\%) because it was found to be simple, requires little training to use and easy to learn. The respondents were also confident that the approach was easy to understand (80) because it used simple language, few steps to follow, clear definition of terms and has a systematic follow. It was also established that the approach covered the necessary scope of REPI in SMEs (complete) (82) and this was due to the approach containing the necessary details as well as covered the necessary scope. The results revealed that the approach 
was also applicable to different SME settings (80), the approach can be tailored to different SME environments, being simple to adopt as well as its flexibility. On the other hand $(19 \%)$ of the respondents did not find the approach easy to use, easy to understand, not sufficient enough and not sure about its applicability. These respondents had not participated in any REPI before in their organizations, so this was a learning challenge as well as good feed back to us. However, with time these respondents picked interest in the approach and are trying it out, and providing positive feedback.

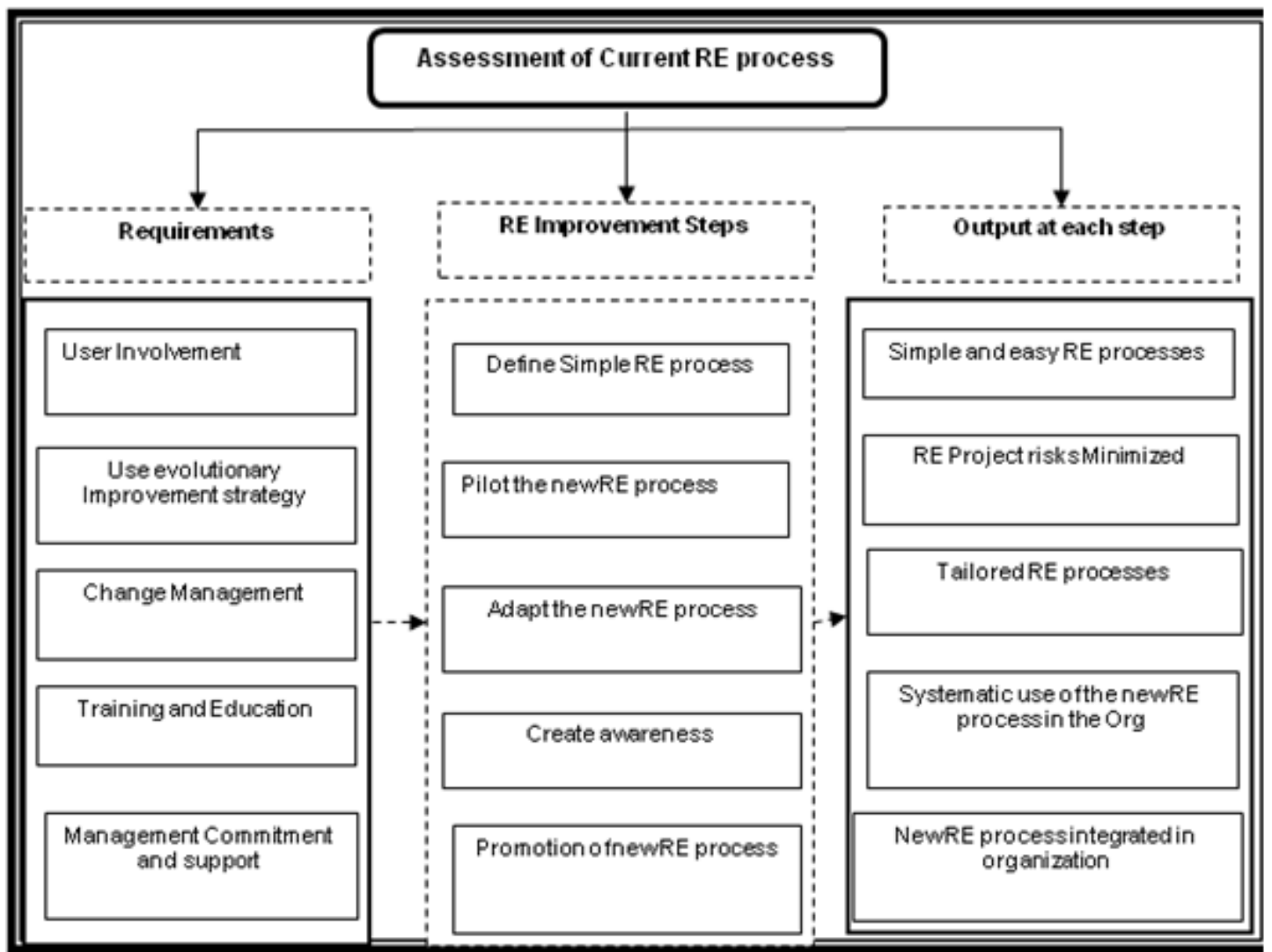

Figure 1; Systematic Approach to RE Process Improvement (SAREPI)

\section{CONCLUSION AND FUTURE RESEARCH}

In this study we looked at the validation of the Systematic approach (SAREPI) to requirements engineering process improvement in SMEs in Uganda. We set out a recommended design science value validation criteria [6] of ease of use, understandability, simplicity and completeness. From the validation results it was established that the approach can be beneficial in REPI and has the following characteristics: easy to use, understandable, complete, simple, applicable and systematic. Therefore suitable and applicable to SMEs in different environments that are in need of REPI. Even when the approach was validated, there is still need to carry out more empirical case studies in different SMEs settings to gain more insights on the different benefits that can be got by SMEs in the different environments.

\section{REFERENCES}

[1] Beecham, S., Hall, T., Britton, C., Cotte, M. and Rainer, A. (2005). Using an expert panel to validate a requirements process improvement model Journal of System and Software 76(3).

[2] Beecham, S., Hall, T., Rainer, A. (2003). Building a requirements process improvement model, Technical Report No. 378 of the department of Computer Science, Faculty of Engineering and Information Sciences. University of Hertfordshire, Centre for Empirical Software Process Research.
[3] Damian, D., Zowghi, D., Vaidyanathasamy, L., and YogendraPal. (2003). An industrial case study of immediate benefits of requirements engineering process improvement at the Australian Center for Unisys Software. Empirical Software Engineering Journal.

[4] Dominic, T. (2009). Seven steps to achieving better requirements engineering in your organization IBM. Requirements engineering to support your business objects. Rational Software.

[5] Gonzalez, Rafael A. (2009). Validation of Crisis Response Simulation within the Design Science Framework. ICIS 2009 Proceedings.

[6] Hakim, C. (1987). Research Design: Strategies and Choices in the Design of Social Research. Contemporary Social Research: 13, ed. M. Bulmer, London: Routledge.

[7] Hevener R.A., March T.S., Park J. and Ram S. (2004). Design Science in information systems Research. Management Information Systems Quarterly.

[8] Kabaale E, Nabukenya J.: A systematic Approach to Requirements Engineering Process Improvement in Small and Medium Enterprises: An Exploratory Study, PROFES 2011

[9] Kamsties, Ormann, K., H, and Schlich, M. (1998). Requirements Engineering in Small and Medium Enterprises: State-of-the-Practice, Problems, Solutions, and Technology Transfer. Published at a conference on 
European Industrial Requirements Engineering (CEIRE'98) London, UK.

[10] Kauppinen, M., Vartiainen, M., Kontio, J., Kujala, S., Sulonen, R. (2004). Implementing requirements engineering processes throughout organizations: success factors and challenges. Information and Software Technology 46 (937-953)

[11] Mishra, D and Mishra, A. (2009) SoftwaREPI in SMEs: A Comparative View. ComSIS 6 (1)

[12] Niazi, M., K. (2002) Improving the Requirements Engineering Process through the Application of a Key Process Areas Approach. Australia Workshop on requirements engineering.

[13] Nikula, U., Sajaniemi, J., and Kälviäinen, H. (2000) A State-of-the-Practice Survey on Requirements Engineering in Small- and Medium-Sized Enterprises. Telecom Business Research Center Lappeenranta. Research Report
[14] Olsson, T., Doerr, J., Koenig, T., and Ehresmann, M. (2005). A Flexible and Pragmatic Requirements Engineering Framework for SME. Proceedings of SREP'05, Paris, France.

[15] Peffers, K., Tuunanen, T., Rothenberger, M., A and Chatterjee, S. (2007). A Design Science Research Methodology for Information Systems Research Published in Journal of Management Information Systems, Volume 24 Issue 3.

[16] Sawyer, P., Sommerville, I., and Viller, S. (1997). Requirements Process Improvement through the Phased Introduction of Good Practice. SoftwaREPI and Practice.

[17] Sommerville, I. and Ransom, J. (2005). An Empirical Study of Industrial Requirements Engineering Process Assessment and Improvement. ACM Transactions on Software Engineering and Methodology, 13(1), 85-117

[18] Wohlin, C., Gustavsson, A., Höst, M, and C. Mattsson. (1996). A Framework for Technology Introduction in Software Organizations, Proceedings SoftwaREP 